\title{
Sequences of epicuticular wax structures along stems in four selected tree species
}

\section{Dominik Tomaszewski* \& Jerzy Zieliński}

Polish Academy of Sciences, Institute of Dendrology, Parkowa 5, 62-035 Kórnik, Poland

* corresponding author (e-mail: dominito@man.poznan.pl)

\begin{abstract}
Wax layer formation accompanies the processes of epidermis and cuticle formation. To examine these changes, observations along current-year long shoots of four woody species (Acer negundo, A. rufinerve, Gymnocladus dioica, and Gingko biloba) were made. Long shoots are suitable objects for such observations, because from the same stem, several samples can be obtained that represent a well-defined sequence of fragments of different ages.

The data that were obtained from different and sometimes distant stem regions suggest that epicuticular wax crystals appear on the stems very early and quickly, since they are found near the stem apex during intensive plant growth. This was confirmed for the four examined species. Some differences, however, were noted.

Very rapid production of prominent wax crystals was observed in stems. In an experiment involving mechanical wax removal from the $A$. negundo stem surface and subsequent analysis of the surface after several days via scanning electron microscopy (SEM), it was shown that wax regenerates very quickly. After only several hours, new emerging crystals were observed and their morphologies were essentially the same. Nearly full regeneration was observed several days after removal.
\end{abstract}

Key words: epicuticular waxes; wax regeneration; stems; SEM

\section{Introduction}

Land plants are especially exposed to unfavourable conditions and factors, such as drought, pathogens (mainly insects and fungi), harmful sunlight, rainfall, dust, and high temperature. For this reason, they developed their own characteristic forms of protection. One form is a wax layer that covers the epidermis of their aboveground organs. The wax layer may be formed as a simple, thin film; consequently, the surfaces of the organs are generally smooth and glossy. In many plant species, however, wax crystals appear on this layer, which makes the surface somewhat matte. Cuticular waxes have been studied for many years with respect to their morphology, chemistry, and functions, mainly protective ones (see Riederer \& Müller 2006). Studies have focused almost exclusively on epicuticular wax on leaves, and information about wax layer covering the stem epidermis has been fragmentary and insufficient. The studies we have performed during the past years indicate that the stems are quite autonomous in these aspects. In many cases, wax structures on stems differ from those on the leaves. Moreover, wax crystals often have different orientation, which is related to the elongation of the stem (Tomaszewski \& Zieliński 2014). Wax layer formation accompanies the processes of epidermis and cuticle formation. In the case of a smooth wax layer, its formation is practically impossible to observe via SEM. For wax layer with crystals, the changes become visible when on the smooth surface more or less remarkable crystalline structures appear. Few works exist that report the stages of these characteristic wax structures on leaves (Koch et al. 2004). To our knowledge, there are no such observations for stems. To examine these changes, observations along current-year long shoots of four woody species were made. Long shoots are suitable objects for such observations, because from the same stem, several samples can be obtained that represent well-defined sequences of fragments of different age.

Epicuticular waxes can be mechanically damaged quite easily, especially their crystals. There are reports published that provide data regarding good and fast regeneration of wax layer on leaves using atomic force 
microscopy (Koch et al. 2004, 2009). Since our goal was to examine regeneration of wax crystals on stems, we performed an experiment that consisted in removing epicuticular wax crystals from current-year waxy long shoots in Acer negundo.

The goal of our study was to investigate the alongstem sequence of external wax layer formation regarding the age of the stem fragment. Use of SEM does not allow observation of the surface of living plants; therefore, an analysis of changes in time in the same place was not possible. It is very likely, however, that a sequence of changes along a stem reflects changes in one place or corresponds with them. The sequence started from the top of the stem and ended at its base.

The results generated from analysing wax structures' morphology along stems may be useful for selecting a place from which material for comparative studies should be taken. For this reason, a description of variability along a stem is needed.

\section{Material and methods}

Series of micrographs of Acer negundo L., A. rufinerve Siebold \& Zucc. (Sapindaceae), Gymnocladus dioica (L.) K. Koch (Fabaceae), and Gingko biloba L. (Gingkoaceae) were analysed. The stems were sampled from the Kórnik Arboretum on 29 July 2012 (A. negundo), 11 June 2012 (A. rufinerve), 18 June 2012 ( $G$. dioica), and 11 June and 30 July 2012 (G. biloba).

Epidermal surface was examined using SEM. The stem fragments were sampled from several places along a single stem. The samples were mounted on aluminium stubs using conductive carbon adhesive tabs and air-dried under ambient conditions. They were then coated with gold and viewed with a Hitachi S3000N SEM equipped with a secondary electron detector; the accelerating voltage used was $15 \mathrm{kV}$. Images were taken at different magnifications. In total, more than 300 images were analysed.

For observation of wax regeneration, mechanical removal of wax was performed on several current-year stems of a selected individual of $A$. negundo in the field. Epidermis fragments were sampled immediately after removal, $2 \mathrm{~d}$, and $7 \mathrm{~d}$, each instance from a different stem. The samples were observed in SEM using the same protocol as described above.

For classification of wax 3-D crystals forms, the system proposed by Barthlott et al. (1998) was used.
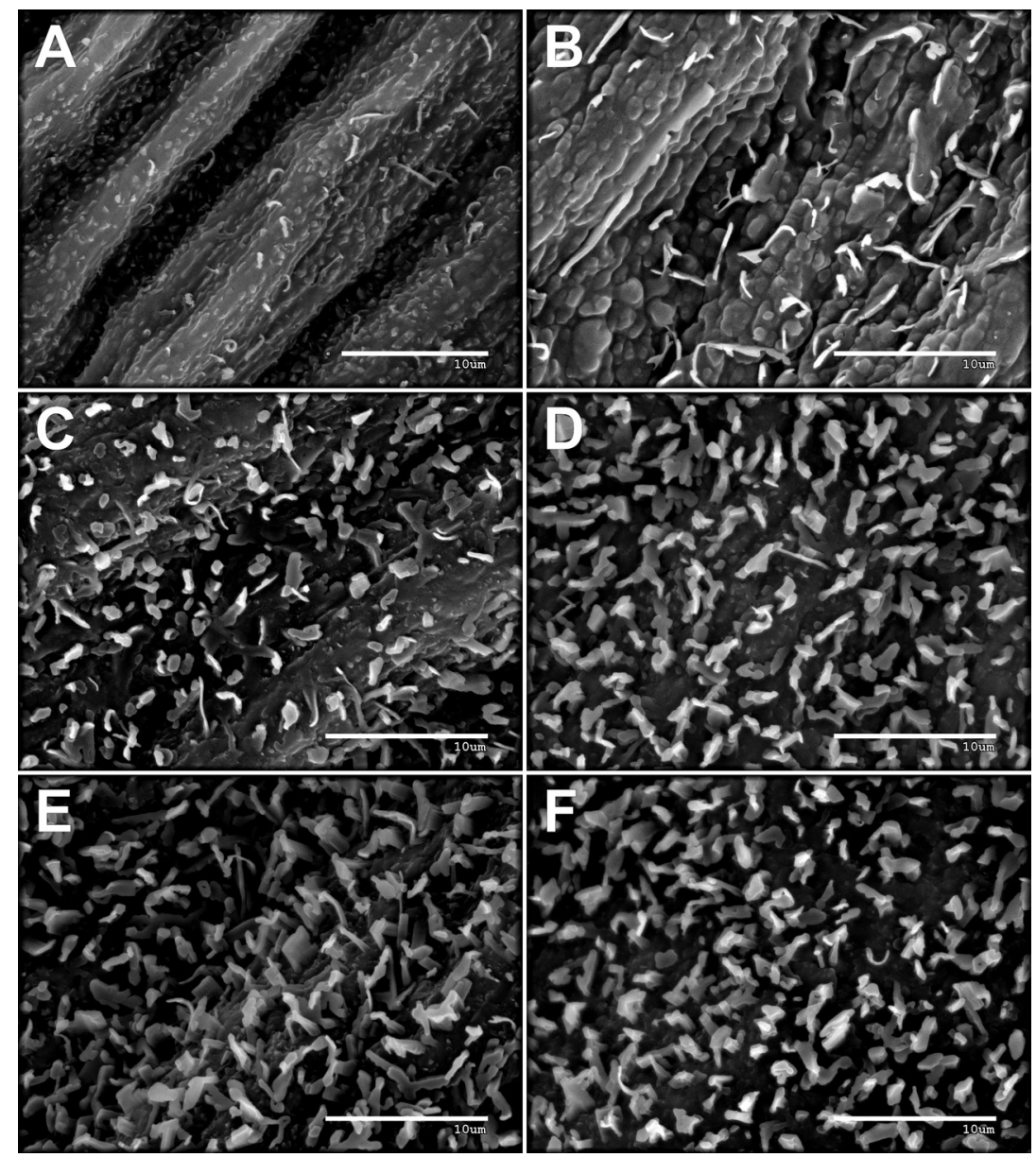

Fig. 1. Wax crystals in different regions along a stem of Gymnocladus dioica

Explanations: A $-5 \mathrm{~cm}$ from the top of the stem, B $-10 \mathrm{~cm}, \mathrm{C}-23 \mathrm{~cm}, \mathrm{D}-31 \mathrm{~cm}, \mathrm{E}-41 \mathrm{~cm}, \mathrm{~F}-57 \mathrm{~cm}$ (scale bars $=10 \mu \mathrm{m})$ 


\section{Results and discussion}

On stem surface of Gymnocladus dioica, wax crystals were found that could be classified as transitional forms between polygonal rodlets, transversely ridged rodlets, and platelets. However, these structures likely belong to just one type, which has not yet been recognised.

Fig. 1 shows surfaces of different stem zones in G. dioica. The first micrograph (Fig. 1A) shows the youngest part of the stem, near its top. The epidermis in this region was not yet fully developed, but some roughness and very small wax structures could be already observed. The forms of these structures allow their classification as elongated platelets.

Below the youngest part of the stem (Fig. 1B), platelets were also present. However, they were significantly larger. Other wax crystals were also present there; they seemed to be platelets with irregular edges. They would likely become larger polygonal rodlets. On the basis of the micrographs from the lower parts of the stem, it may be presumed that the stem surface does not show differences in terms of the wax crystals there (Figs. 1CF). After the initial stages, no further changes occurred in the epidermis, or if so, they were not pronounced at the structural level.

In $A$. negundo, the stem surface was covered with crystals that shared characteristics of both platelets and rodlets. They tended to coil and even formed typical tubular structures. Also, the wax structures showed zonal character, which likely reflects the sequence of their development on the cuticle surface (Figs. 2A and 2B). The structures were initially formed as scattered, small, and coiled rodlets or platelets. They increased in size quite rapidly, as the micrographs taken from the location $2.5 \mathrm{~cm}$ below the top of the stem showed the same crystal structures that became much larger and more complex. Figs. 2C-D were taken from the lower parts of the stem. Crystals were of the same type; however, they were more densely distributed on the cuticle surface.

Micrographs from very distant lower parts of the stem (Fig. 2E-F) show wax structures formed the same year but much earlier. They differed in shape and size from the structures found above and could be classified as coiled rodlets. They may be either a result of erosion of the structures described from the upper stem parts, or the epidermis in A. negundo produces different wax
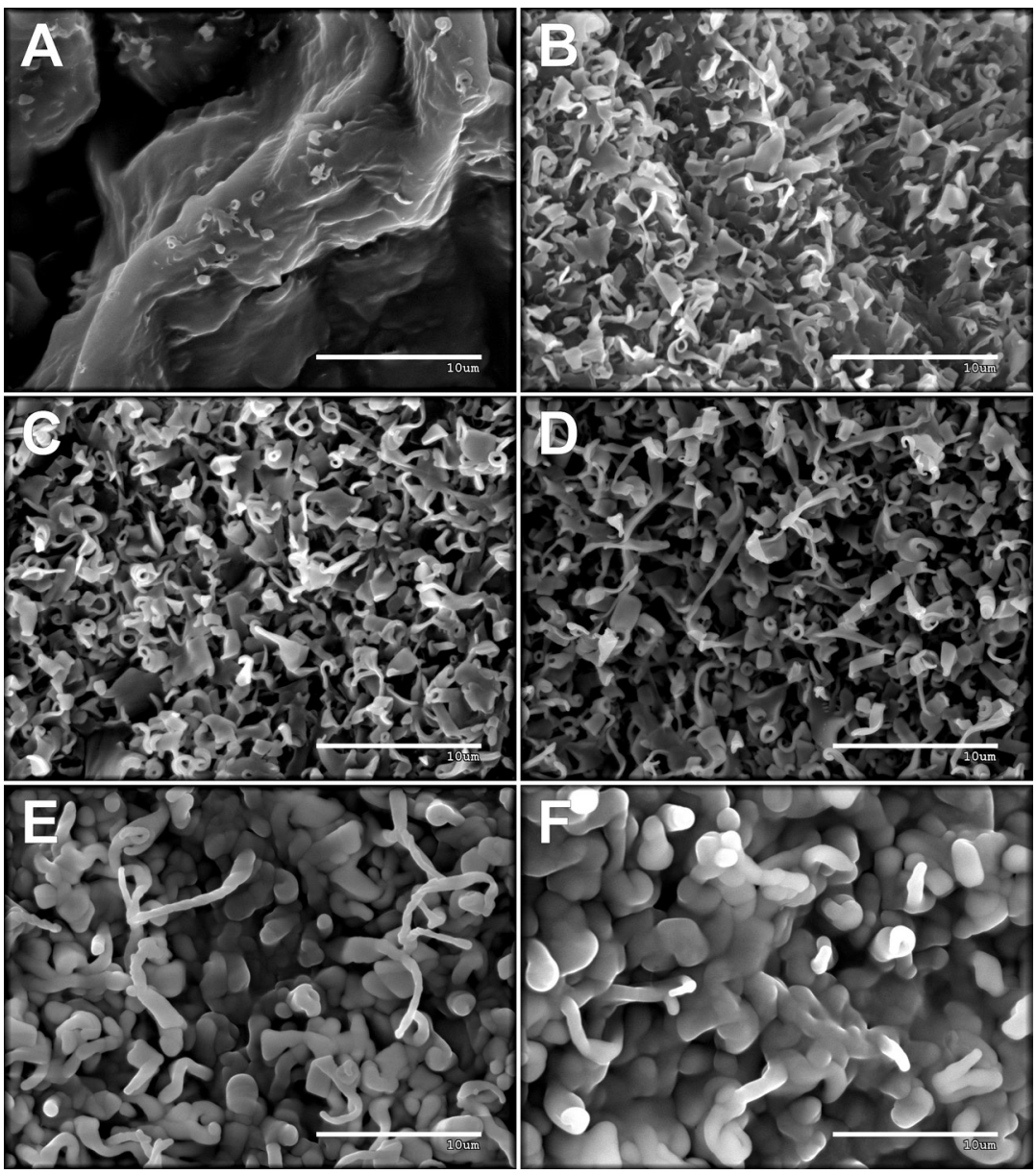

Fig. 2. Wax crystals in different regions along a stem of Acer negundo

Explanations: A $-0.5 \mathrm{~cm}$ from the top of the stem, B $-2.5 \mathrm{~cm}, \mathrm{C}-6.5 \mathrm{~cm}, \mathrm{D}-28 \mathrm{~cm}, \mathrm{E}-46 \mathrm{~cm}, \mathrm{~F}-76 \mathrm{~cm}$ (scale bars $=10 \mu \mathrm{m}$ ) 


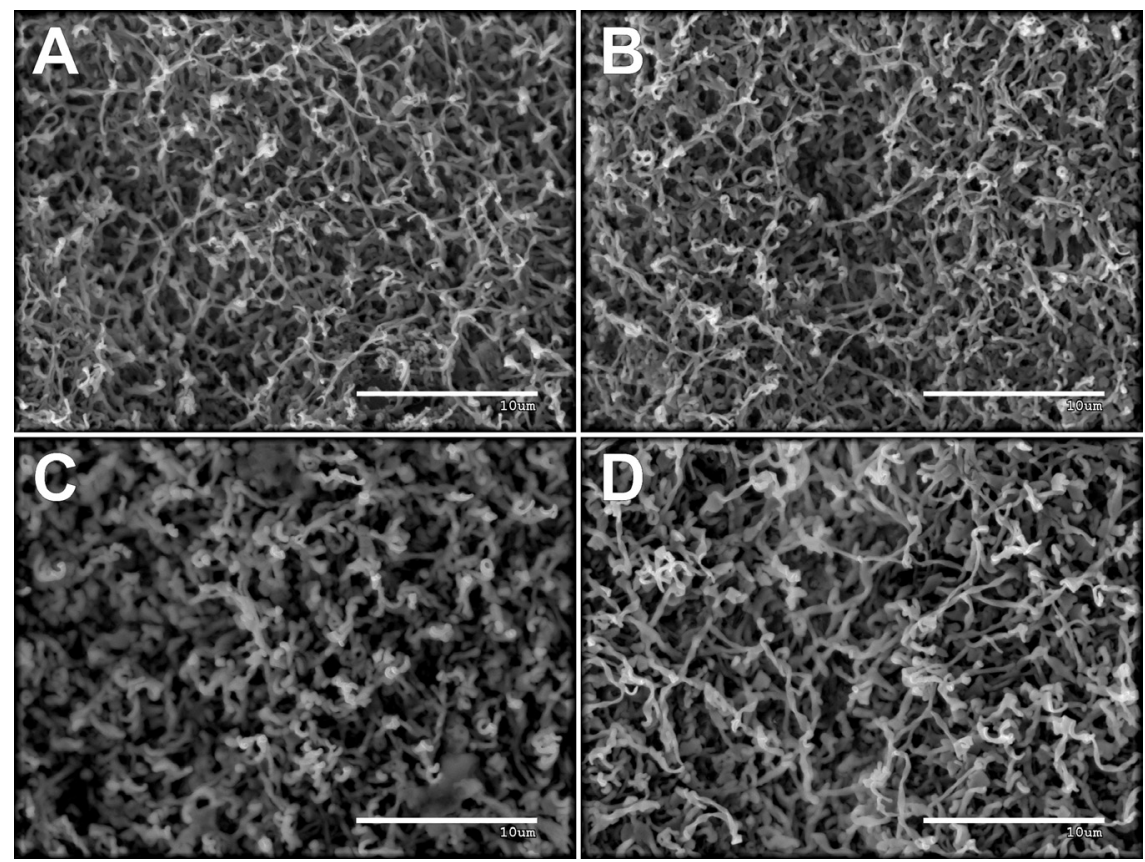

Fig. 3. Wax crystals in different regions along a stem of Acer rufinerve

Explanations: A -1.5 from the top of the stem, B $-2.5 \mathrm{~cm}, \mathrm{C}-5.5 \mathrm{~cm}, \mathrm{D}-11.5 \mathrm{~cm}$ (scale bars $=10 \mu \mathrm{m})$
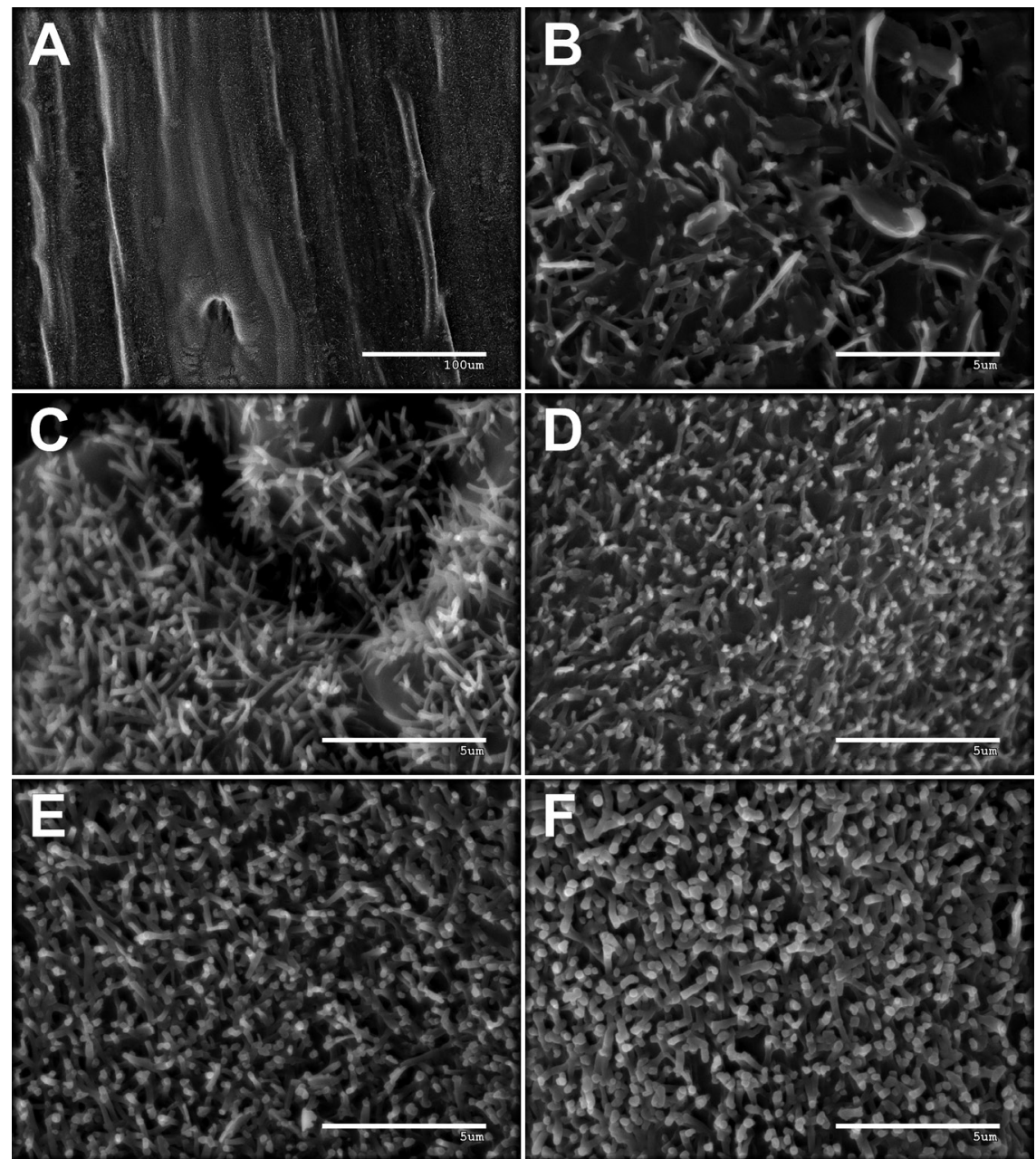

Fig. 4. Wax crystals in different regions along a stem of Ginkgo biloba

Explanations: A $-14 \mathrm{~cm}$ from the top of the stem (zones with higher density of tubules around the stoma, transitional forms between tubules and platelets are found farther from it), B $-14 \mathrm{~cm}$ (tubules and transitional forms), $\mathrm{C}-$ near the top of the stem, $\mathrm{D}-3 \mathrm{~cm}, \mathrm{E}-14 \mathrm{~cm}, \mathrm{~F}-22 \mathrm{~cm}$ (scale bars A=100 $\mu \mathrm{m}$; $\mathrm{B}-\mathrm{F}=5 \mu \mathrm{m})$ 


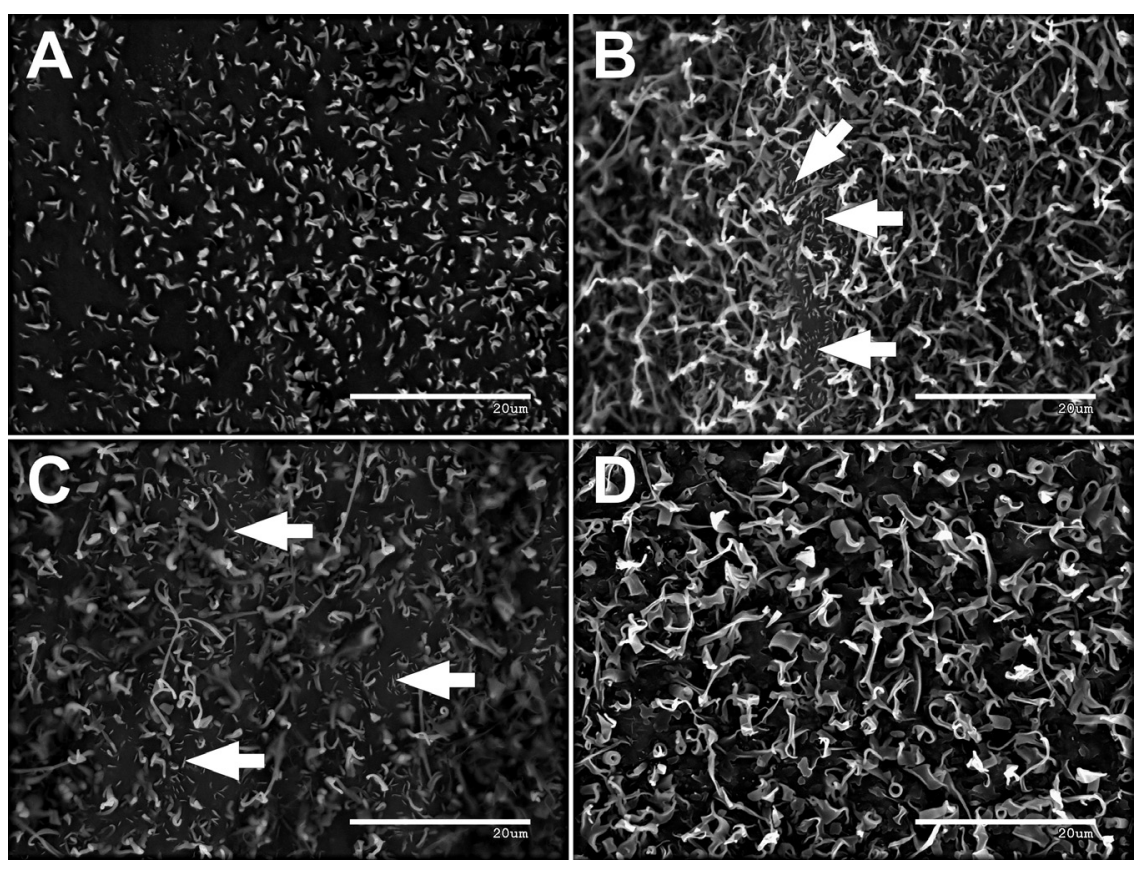

Fig. 5. Wax crystal regeneration on current-year stems of Acer negundo after mechanical removal Explanations: A - after several hours, B, C - after $2 \mathrm{~d}$ (arrows indicate platelets as regeneration wax types), D - after $7 \mathrm{~d}($ scale bars $=20 \mu \mathrm{m})$

crystals during the same year. It would be interesting to investigate whether this is linked to differences and changes in chemistry of the wax crystals along the stem.

Micrographs of A. rufinerve (Fig. 3) do not show any substantial variability in wax crystal formations along its stem. In this species, the stem was covered with interwoven coiled rodlets, forming a net. The lack of visible differences may indicate that its epidermis only $1.5 \mathrm{~cm}$ below the stem apex is characterised by fully developed epicuticular wax crystals.

In Ginkgo biloba, two types of zonal arrangement in wax crystals on stems existed. The first one is related to variability of wax layer formation along the stem. This may be a result of epidermis growth and development (younger cells on the top vs. older cells below). The second arrangement of wax crystals (crosswise) is linked to the distance from the stomatal apparatus. In many plants, perhaps in the majority, stomata are found not only on leaves but also on stems; G. biloba is one of these plant species (Fig. 4A). It is known that in many plant species, wax structures can vary when they are located near stomata. This is especially clear in many gymnosperms. For example, in Pinus sylvestris, the wax crystals (tubules) are concentrated in rows of stomata; they are virtually absent or scarce in other regions of the needle surface. In G. biloba, the zonal crosswise distribution results from the fact that near stomata, dense tubules were found. When the distance from the stomata increases, the tubules become less numerous, and other structures may appear (platelet-like or polymorphic structures that are transitional between platelets and tubules (Fig. 4B).

Similarly to other species, wax crystals in G. biloba quickly appear on the cuticle. Well-formed platelets and relatively few tubules were observed on the top of the stem (just below its apex, Fig. 4C). In lower regions, the tubules were more frequent. Zones of stripes, perpendicular to the long stem axis, may appear where the wax crystals are less dense or absent. This suggests that crystals were formed there before the intensive stem elongation and that they have been driven apart due to sufficient cell elongation.

The data obtained from the different and sometimes distant stem regions suggest that epicuticular wax crystals appear on the stem very early and quickly, since they are found near the stem apex during intensive growth of a plant. This was confirmed for all four examined species. Some differences, however, were noted.

Very rapid production of both prominent wax crystals and smooth wax layer covering the leaf cuticle was previously noted in selected plant species (Koch et al. 2004 , 2009). Rapid production was observed in stems, as well. In this experiment that consisted in mechanical wax removal from the $A$. negundo stem surface and SEM analysis of the surface several days later, it was shown that wax regenerates very quickly. New, emerging crystals were observed after only several hours, and the morphology of the crystals was essentially identical. 
Nearly full regeneration was observed several days after removal. However, small platelets were also found, which were absent on primary undamaged surfaces (Fig. 5B-C).
Acknowledgments. This study was funded by the Ministry of Science and Higher Education and the National Science Centre (Project No. N N303615338) during 2010-2013. We are very grateful to Mrs. Magdalena Gawlak (Institute of Plant Protection, National Research Institute, Poznań, Poland) for her excellent technical assistance.

\section{References}

Barthlott W., Neinhuis C., Cutler D., Ditsch F., Meusel I., Theisen I. \& WiLheLmi H. 1998. Classification and terminology of plant epicuticular waxes. Bot. J. Linn. Soc. 126: 237-260. DOI: 10.1111/j.1095-8339.1998. tb02529. $x$

Koch K., Bhushan B., Ensikat H.-J. \& Barthlott W. 2009. Self-healing of voids in the wax coating on plant surfaces. Philos. Transact. A Math. Phys. Eng. Sci. 367: 1673-1688. DOI: 10.1098/rsta.2009.0015

Koch K., Neinhuis C., Ensikat H. J. \& Barthlott W. 2004. Self assembly of epicuticular waxes on living plant surfaces imaged by atomic force microscopy (AFM). J. Exp. Bot. 55: 711-718. DOI: 10.1093/jxb/erh077 Riederer M. \& Müller C. (eds.). 2006. Biology of the Plant Cuticle. 438 pp. Blackwell Publishing, Oxford.

ToMAsZewSKi D. \& ZIELIŃSKI J. 2014. Epicuticular wax structures on stems and comparison between stems and leaves -A survey. Flora 209: 215-232. DOI: 10.1016/j. flora.2014.03.001 